\section{(2) OPEN ACCESS}

\title{
Diagnostic performance of an acoustic-based system for coronary artery disease risk stratification
}

\author{
Simon Winther, ${ }^{1}$ Louise Nissen, ${ }^{2}$ Samuel Emil Schmidt, ${ }^{3}$ Jelmer Sybren Westra, ${ }^{1}$ \\ Laust Dupont Rasmussen, ${ }^{2}$ Lars Lyhne Knudsen, ${ }^{2}$ Lene Helleskov Madsen, ${ }^{2}$ \\ Jane Kirk Johansen, ${ }^{4}$ Bjarke Skogstad Larsen, ${ }^{3}$ Johannes Jan Struijk, ${ }^{3}$ Lars Frost, ${ }^{4}$ \\ Niels Ramsing Holm, ${ }^{1}$ Evald Høj Christiansen, ${ }^{1}$ Hans Erik Botker, ${ }^{1}$ Morten Bøttcher ${ }^{2}$
}

\begin{abstract}
- Additional material is published online only. To view please visit the journal online (http://dx.doi.org/10.1136/ heartjnl-2017-311944).
\end{abstract}

${ }^{1}$ Department of Cardiology, Aarhus University Hospital, Aarhus, Denmark

${ }^{2}$ Department of Cardiology, Hospital Unit West, Herning, Denmark

${ }^{3}$ Department of Health Science and Technology, Aalborg University, Aalborg, Denmark ${ }^{4}$ Department of Cardiology, Regional Hospital of Silkeborg, Silkeborg, Denmark

Correspondence to Dr Simon Winther, Department of Cardiology, Aarhus University Hospital, Palle Juul-Jensens Boulevard 99, DK-8200 Aarhus, Denmark; sw@dadlnet.dk

Received 6 June 2017 Revised 17 October 2017 Accepted 17 October 2017 Published Online First 9 November 2017
Check for updates

To cite: Winther S, Nissen L, Schmidt SE, et al. Heart 2018;104:928-935.

\section{ABSTRACT}

Objective Diagnosing coronary artery disease (CAD) continues to require substantial healthcare resources. Acoustic analysis of transcutaneous heart sounds of cardiac movement and intracoronary turbulence due to obstructive coronary disease could potentially change this. The aim of this study was thus to test the diagnostic accuracy of a new portable acoustic device for detection of CAD.

Methods We included 1675 patients consecutively with low to intermediate likelihood of CAD who had been referred for cardiac CT angiography. If significant obstruction was suspected in any coronary segment, patients were referred to invasive angiography and fractional flow reserve (FFR) assessment. Heart sound analysis was performed in all patients. A predefined acoustic CAD-score algorithm was evaluated; subsequently, we developed and validated an updated CAD-score algorithm that included both acoustic features and clinical risk factors. Low risk is indicated by a CADscore value $\leq 20$.

Results Haemodynamically significant CAD assessed from FFR was present in $145(10.0 \%)$ patients. In the entire cohort, the predefined CAD-score had a sensitivity of $63 \%$ and a specificity of $44 \%$. In total, $50 \%$ had an updated CAD-score value $\leq 20$. At this cut-off, sensitivity was $81 \%$ (95\% Cl 73\% to $87 \%$ ), specificity $53 \%$ (95\% Cl $50 \%$ to $56 \%$ ), positive predictive value $16 \%$ (95\% $\mathrm{Cl} 13 \%$ to $18 \%$ ) and negative predictive value $96 \%$ (95\% Cl 95\% to 98\%) for diagnosing haemodynamically significant CAD.

Conclusion Sound-based detection of CAD enables risk stratification superior to clinical risk scores. With a negative predictive value of $96 \%$, this new acoustic rule-out system could potentially supplement clinical assessment to guide decisions on the need for further diagnostic investigation.

Trial registration number ClinicalTrials.gov identifier NCT02264717; Results.

\section{INTRODUCTION}

Diagnosing patients with symptoms suggestive of obstructive coronary artery disease (CAD) continues to require substantial healthcare resources. Despite several attempts to improve clinical pretest risk scoring algorithms for obstructive $\mathrm{CAD}$, the incidence of a normal diagnostic test remains high. ${ }^{1}$ A continued high rate of inappropriate Invasive coronary angiography (ICA) in de novo patients has been criticised in particular because it is costly and carries the risk of complications. Hence, there is a pertinent need for more accurate risk stratification tools providing high negative predictive value (NPV) to accurately rule out obstructive $\mathrm{CAD}$ to avoid further diagnostic testing. $^{3}$

One plausible way to obtain this goal may be to use advanced analysis of sounds originating from blood flow turbulence in the coronary circulation during diastole. Acoustic detection of obstructive CAD is non-invasive, radiation free and economically favourable. This makes the technique attractive for primary risk stratification. Recent advances in sound sensor technology, analytic power and data filtering have enabled the use of acoustic detection to diagnose intracoronary turbulence due to obstructive CAD. In addition to weak murmurs related to intracoronary turbulence recent approaches have analysed further aspects of the heart sounds which might relate to myocardial compliance and resonance frequencies of the coronary artery tree. ${ }^{4-7}$ Several methodological studies describe the frequency range and timing of turbulence associated with obstructive CAD, and several companies have developed European Conformity marked equipment with different algorithms to diagnose obstructive CAD. ${ }^{8}$ These developments have paved the way for investigation of the technique in clinical practice. ${ }^{589}$ Hence, small-scale pilot studies of different acoustic devices have demonstrated NPVs in the range 71\%-98\% suggesting that they may be used as rule-out modalities. ${ }^{4011}$

In the present study of acoustic detection of CAD, we aimed to test an acoustic device in a low to intermediate risk population using both quantitative coronary angiography (QCA) and invasive fractional flow reserve (FFR) as references. Two algorithms were tested: a previously used acoustic algorithm, CAD-score Version 2 (V2) (per protocol), which was developed using patients included in pilot studies; and a new algorithm, CAD-score V3, by combining an updated acoustic algorithm and clinical risk factors.

\section{MATERIALS AND METHODS}

\section{Study design and population}

The present study is a predefined substudy of the Danish study of Non-Invasive testing in Coronary Artery Disease (Dan-NICAD). Patients referred to cardiac CT angiography (CTA) as first-line test due 


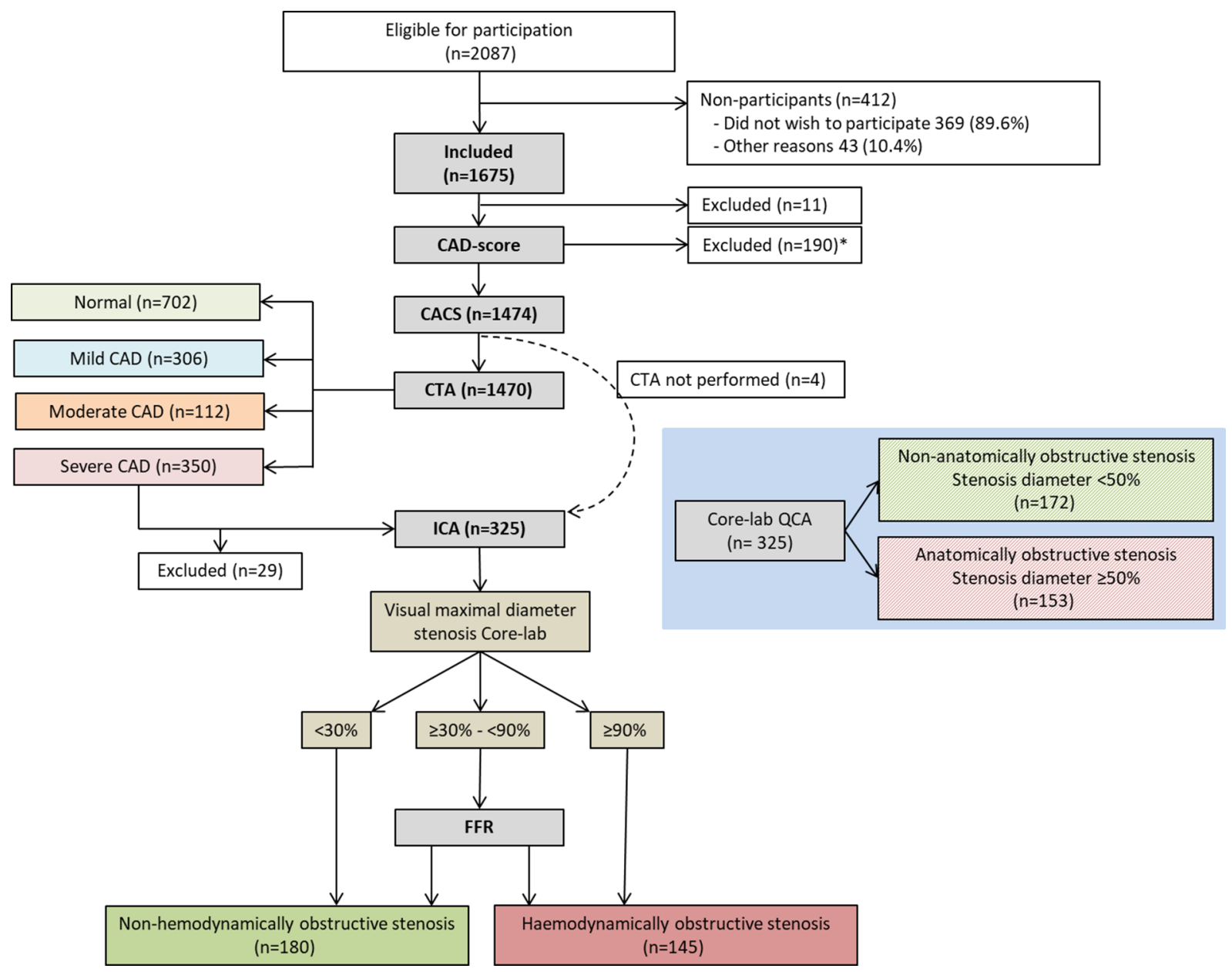

Figure 1 Flow chart of patients in the study for final diagnosis of CAD. *A CAD-score Version 2 was not calculated in 190 patients and CAD-score Version 3 was not calculated in 204 patients. Median intertest interval from the cardiac CTA to the ICA was 30 days (10th and 90th percentiles: 14 and 50 days). CACS, coronary artery calcium score; CAD, coronary artery disease; CAG, invasive coronary angiography; CTA, CT angiography; FFR, fractional flow reserve; ICA, invasive coronary angiograph; QCA, quantitative coronary angiography.

to a history of symptoms suggestive of obstructive CAD were consecutively enrolled. Referral decision was taken during an outpatient visit by a cardiologist based on echocardiography, patient history and risk profiling according to national and European Society of Cardiology guidelines. ${ }^{12}$ Exclusion criteria were: (1) age $<40$; (2) previous coronary revascularisation; (3) estimated glomerular filtration $<40 \mathrm{~mL} / \mathrm{min}$; (4) pregnancy; and (5) contraindication for iodine-containing contrast medium, MRI or adenosine (severe asthma, advanced atrioventricular block or critical aorta stenosis). All patients signed a written informed consent form.

All patients underwent a systematic interview to assess risk factors and symptoms to calculate the updated Diamond-Forrester score (DF score). ${ }^{13}$ In connection with the cardiac CTA, examination was performed with the acoustic CADScor ${ }^{\circledR}$ System (Acarix A/S, Denmark). Patients with suspicion of a coronary stenosis at cardiac CTA underwent ICA with FFR. Patients without stenosis at cardiac CTA were terminated from the study (figure 1). The study design, imaging protocols and analysis strategy have been published. ${ }^{14}$

\section{CAD-score}

Heart sound recordings were obtained transcutaneously with the CADScor ${ }^{\circledR}$ System (Acarix $\mathrm{A} / \mathrm{S}$ ) microphone mounted at the fourth intercostal space just to the left of the sternum using a dedicated adhesive patch. The patient lied down for $3 \mathrm{~min}$ during the recording and was asked to hold his/her breath four times, each time for $8 \mathrm{~s}$ (figure 2). As the equipment may be influenced by excessive noise, the tests were conducted in an undisturbed room. The CAD-score was estimated immediately after the recording with a fully automated and device embedded algorithm. $^{45915}$ If the recording quality was insufficient the recording device requested a new recording. In these cases, a second attempt was made to calculate the CAD-score. The algorithm combines four measures (Features) of acoustic properties from the diastolic heart sounds which relate to murmurs and other heart sound characteristics into a score (figure 2 and online supplementary material).

An updated score, using the CAD-score V3 algorithm, was calculated using postprocessing of the audio recordings obtained with the recording device. The updated algorithm measures eight acoustic properties and the resulting acoustic score was combined with the clinical risk factors gender, age and hypertension, defined as systolic blood pressure $\geq 140 \mathrm{~mm} \mathrm{Hg}$ or receiving antihypertension medicine (figure 2 and online supplementary material). The updated CAD-score V3 algorithm was developed using a heart sound database (Acarix A/S) with recordings from patients included in previous pilot studies, published data, and a training cohort of the first 593 consecutively enrolled patients from the present Dan-NICAD study. ${ }^{4}$ The remaining 


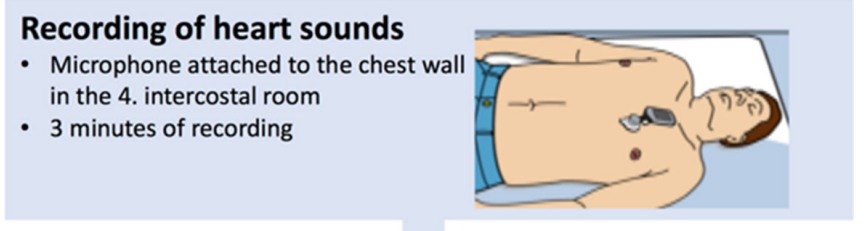

Pre-processing of heart sound recordings:

- Segmentation of the recording into systoles and diastoles.

- Alignment of the heart sounds to S2

- Identification of the mid diastolic period

- Filter the signal
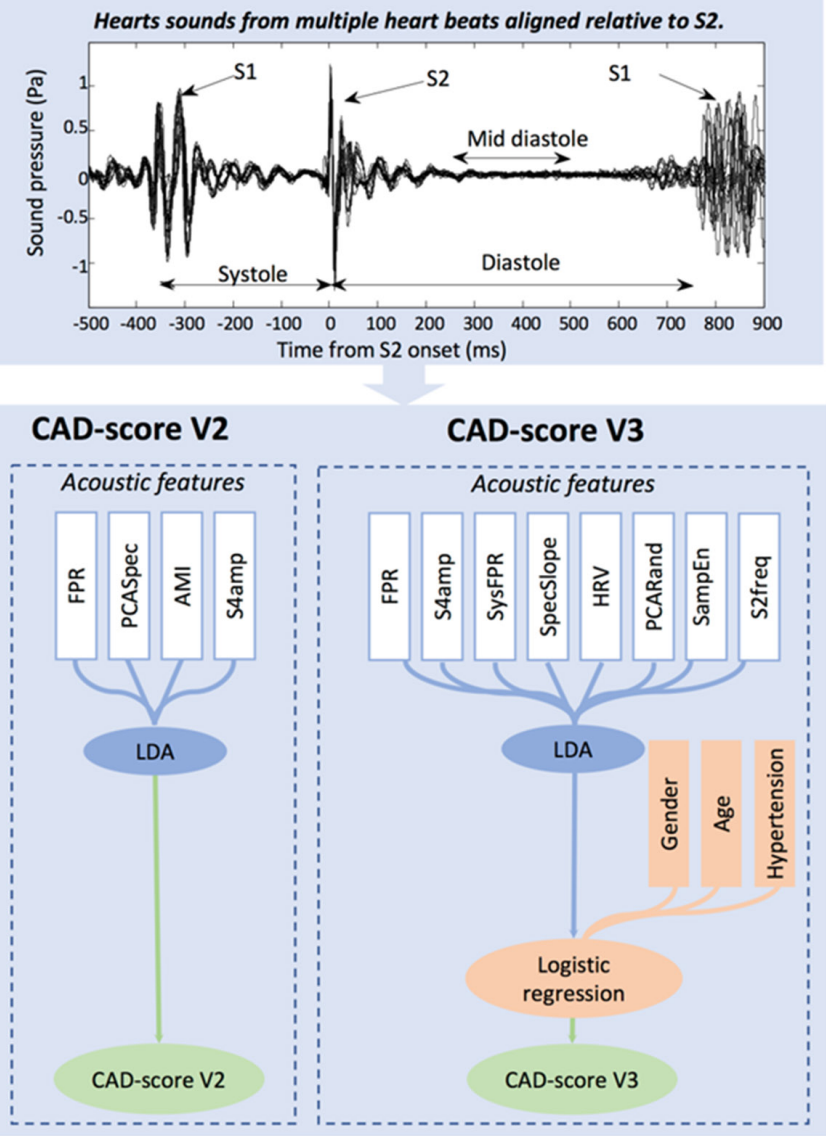

Figure 2 Depiction of the CAD-score acquisition and the two CAD-score algorithms. The heart sounds were recorded at the fourth intercostal space with patients in a supine position. The preprocessing part of the algorithm organised the heart sounds for analysis through segmentation and filtering. After preprocessing, the acoustic features were extracted from the sounds and an acoustic score was constructed using LDA. In CAD-score Version 3 (V3), the acoustic score was further combined with gender, age and hypertension using logistic regression. $\mathrm{AMI}$, automutal information; FPR, frequency power ratio; HRV, heart rate variability; LDA, linear discriminant analysis; PCARand, principle component analyses-based measure of the randomness; PCASpec, principle component analysis of the diastolic frequency spectrum; S2freq, frequency distribution of the second heart sounds; S4amp, amplitude of the fourth heart sound; SampEn, sample entropy; SpecSlope, slope of diastolic frequency spectrum; SysFPR, systolic frequency power ratio.

1082 patients in the Dan-NICAD study were used as a validation cohort and remained blinded to the developer of the CAD-score V3 algorithm.

\section{Coronary CTA}

All cardiac CTA scans were performed using a 320-slice volume CT scanner (Aquilion One, Toshiba Medical Systems, Japan) according to clinical guidelines. CT imaging analysis included an Agatston calcium score and evaluation of luminal diameter stenosis estimation in each segment of the coronary tree using an 18-segment model. Coronary lesions were evaluated blinded to the invasive angiographic findings by an experienced cardiologist. Stenosis severity was classified in all segments with a reference vessel diameter $>2 \mathrm{~mm}$ as no stenosis: $0 \%$ diameter reduction; mild stenosis: 1\%-29\% diameter reduction; moderate stenosis: $30 \%-49 \%$ diameter reduction; and severe stenosis: 50\%-100\% diameter reduction. Segments with suspected severe stenosis and non-evaluable segments with a reference vessel diameter $>2 \mathrm{~mm}$ were defined as having obstructive CAD by cardiac CTA. The cardiac CTA was defined as abnormal if obstructive CAD was not ruled out in all coronary segments. CAD severity was further categorised based on the coronary artery calcium score (CACS) and coronary stenosis severity; non-CAD: $\mathrm{CACS}=0$ and no plaque; mild CAD: $C A C S \geq 0$ and plaque with maximal mild stenosis; moderate CAD: CACS $\geq 0$ and plaque with moderate stenosis; and severe $\mathrm{CAD}$ : $\mathrm{CACS} \geq 0$ and plaque with severe stenosis.

\section{Invasive coronary angiography}

The ICA examination was performed according to clinical guidelines. Intracoronary nitroglycerine was administered before the first angiographic acquisition.

QCA analysis was done using QAngioXA V.7.3 software in an independent core lab (ClinFact, Leiden, The Netherlands). Anatomically significant obstructive CAD was defined as a coronary artery with a luminal diameter reduction $\geq 50 \%$ in a segment with a reference vessel diameter $>2 \mathrm{~mm}$.

Invasive FFR was measured in lesions with a visualised, estimated 30\%-90\% diameter stenosis located in vessels with a reference diameter $>2.0 \mathrm{~mm}$ using a clinical FFR system (Aeris, St. Jude Medical, USA). Haemodynamically significant obstructive CAD was identified in a blinded core lab as: (1) FFR value $<0.80$, (2) luminal diameter stenosis reduction $>90 \%$, or (3) luminal diameter stenosis reduction $\geq 50 \%$ if FFR was indicated but not performed for technical, anatomical or other reasons.

\section{Statistical analysis}

Variables are expressed as mean $( \pm \mathrm{SD}$ or total range) or median (range) according to the Gaussian distribution. Categorical variables are reported as frequencies (percentages). The unpaired Student's t-test and analysis of variance test were used for comparison between Gaussian-distributed variables. Wilcoxon rank-sum test and the $\chi^{2}$ test were used for comparison between non-Gaussian distributed and categorical variables, respectively. Pearson's test and Spearman's test were used to analyse correlations of variables of Gaussian and non-Gaussian distributions, respectively. The area under the receiver operating characteristic curve was calculated for continuous variables and compared with the method described by DeLong et al. ${ }^{16}$ The CAD-score was divided as a binary variable with a cut point of $>20$; and sensitivity, specificity, positive predictive value, NPV, and positive and negative likelihood ratios were calculated.

For development of CAD-score V3, the cohort was split in training and validation cohorts according to a prespecified date. Diagnostic accuracy of CAD-score V3 for the training and validation cohort is presented in online supplementary table S2 
and pooled together in the Results section due to similar diagnostic performance in the two cohorts. Statistical analyses were performed using STATA V.13.1 (StataCorp, USA).

\section{RESULTS}

A total of 1675 patients were included. Eleven patients $(0.7 \%)$ were excluded because they did not complete either the CACS or the cardiac CTA. The acoustic CAD-score V2 could not be obtained in 190 patients (11.3\%), and CAD-score V3 was missing in another 14 patients (online supplementary table S1). Of these, four patients $(0.2 \%)$ had missing cardiac CTA data (figure 1).

Baseline and cardiac CTA characteristics are summarised in table 1. Of the patients referred for ICA, 325 patients (91.8\%) completed the investigation. Of these, 153 (47.1\%) were classified with obstructive CAD by core lab QCA, while 145 patients (44.6\%) had haemodynamically obstructive disease based on ICA and FFR (figure 1, table 1). In total, the prevalence of anatomically significant obstructive CAD was $10.6 \%$, and the prevalence of haemodynamically significant obstructive CAD was $10.0 \%$. No adverse events related to the acoustic system were recorded.

\section{CAD-score V2}

The diagnostic accuracy of acoustic CAD-score V2 for diagnosing anatomically obstructive stenosis evaluated by area under the curve (AUC) was $58.1 \%$ (95\% CI 53.6\% to 62.6\%), which was significantly lower than the DF score, 65.9\% (95\% CI $61.2 \%$ to $70.4 \%)(\mathrm{P}<0.01)$. Diagnostic accuracy parameters with both anatomically and haemodynamically obstructive stenosis as references are listed in table 2 . There were no differences in diagnostic accuracy with the use of anatomically versus haemodynamically obstructive stenosis as a reference.

\section{CAD-score V3}

The acoustic part of the CAD-score V3 was higher for men than for women, $25.2 \pm 10.4$ vs $19.3 \pm 9.7(\mathrm{P}<0.001)$. Only a weak correlation was demonstrated with age $(\mathrm{r}=0.11, \mathrm{P}<0.001)$, body mass index $(\mathrm{r}=-0.19, \mathrm{P}<0.001)$, systolic blood pressure $(\mathrm{r}=0.10, \mathrm{P}<0.001)$, diastolic blood pressure $(\mathrm{r}=0.02, \mathrm{P}=0.50)$ and CACS $(r=0.17, \mathrm{P}<0.001)$. In the presence of a coronary stenosis at ICA, a weak correlation with max QCA percentage diameter stenosis was observed $(\mathrm{r}=0.14, \mathrm{P}<0.05)$. Patients with anatomically obstructive stenosis had a significantly higher acoustic CAD-score V3 than patients without, $24.4 \pm 10.2$ vs $21.9 \pm 10.4(\mathrm{P}<0.01)$. The AUC for diagnosing anatomically obstructive stenosis of the acoustic part of the CAD-score V3 was $63.3 \%$ (95\% CI $58.7 \%$ to $67.8 \%)$. This was significantly higher than the previous acoustic CAD-score V2 $(\mathrm{P}<0.05)$, and similar to that of the DF score $(\mathrm{P}=0.40)$.

The final CAD-score V3, which includes both an acoustic part and risk factors, correlated moderately with CACS $(r=0.38$, $\mathrm{P}<0.001)$. The $\mathrm{CAD}$-score $\mathrm{V} 3$ was associated with CAD severity when stratified by (1) CACS groups $(\mathrm{P}<0.001)$, (2) CAD disease severity defined by cardiac CTA $(\mathrm{P}<0.001)$ and $(3)$ obstructive disease $(\mathrm{P}<0.001)$ identified by cardiac CTA (figure 3$)$.

The mean CAD-score V3 was higher for patients with haemodynamically significant CAD than for patients without, $29.9 \pm 12.0$ vs $20.4 \pm 12.0(\mathrm{P}<0.001)$. In patients with a stenosis on ICA, CAD-score V3 correlated with maximal QCA diameter stenosis $(r=0.23, \mathrm{P}<0.001)$ and increased with $\mathrm{QCA}$ diameter stenosis severity $(\mathrm{P}<0.01)$ (online supplementary figure $\mathrm{S} 1$ ). However, no such correlation was present for FFR measurements in patients with $30 \%-90 \%$ diameter stenosis on visual
Table 1 Patient demographics and imaging study characteristics

\begin{tabular}{|c|c|}
\hline \multicolumn{2}{|l|}{ Characteristic $(n=1474)$} \\
\hline Race, Caucasian & $1464(99.3 \%)$ \\
\hline Sex, male & $719(48.8 \%)$ \\
\hline Age (years) & $57.2 \pm 8.8$ \\
\hline Genetic predisposition* & $557(37.8 \%)$ \\
\hline Body mass index $\left(\mathrm{kg} / \mathrm{m}^{2}\right)$ & $26.7 \pm 4.1$ \\
\hline Abdominal circumference $(\mathrm{cm})$ & $93.0 \pm 12.6$ \\
\hline \multicolumn{2}{|l|}{ Blood pressure } \\
\hline Systolic & $138 \pm 19$ \\
\hline Diastolic & $83 \pm 11$ \\
\hline Heart ratet & $65 \pm 11$ \\
\hline \multicolumn{2}{|l|}{ Smoking } \\
\hline Never & $703(47.7 \%)$ \\
\hline Former & $538(36.5 \%)$ \\
\hline Active & $233(15.8 \%)$ \\
\hline Diabetes & $79(5.4 \%)$ \\
\hline \multicolumn{2}{|l|}{ Symptoms $(n=1474)$} \\
\hline Typical chest pain & $410(27.8 \%)$ \\
\hline Atypical chest pain & $495(33.6 \%)$ \\
\hline Non-specific & $569(38.6 \%)$ \\
\hline \multicolumn{2}{|l|}{ CCS Functional Classification of Angina $\geq 2$} \\
\hline 1 (angina only with strenuous exertion) & $1054(71.5 \%)$ \\
\hline$\geq 2$ (angina with moderate or severe exertion) & $113(7.7 \%)$ \\
\hline Updated Diamond-Forrester score & $39 \%(20 \%-54 \%)$ \\
\hline \multicolumn{2}{|l|}{ Risk groups according to updated Diamond-Forrester score } \\
\hline Low risk $(<15 \%)$ & $210(14.2 \%)$ \\
\hline Moderate risk ( $\geq 15 \%$ and $85 \%$ ) & $1229(83.4 \%)$ \\
\hline High risk ( $\geq 85 \%)$ & $35(2.4 \%)$ \\
\hline \multicolumn{2}{|l|}{ Echo $(n=1474)$} \\
\hline Left ventricular ejection fraction & $59.9 \% \pm 3.4$ \\
\hline Cardiac valve disease, any & $71(4.8 \%)$ \\
\hline Aorta insufficiency (mild to moderate) & $36(2.4 \%)$ \\
\hline Other systolic valve disease (mild to moderate) & $35(2.4 \%)$ \\
\hline \multicolumn{2}{|l|}{ Coronary artery calcium score (CACS) $(n=1474)$} \\
\hline Median & $0(0-80)$ \\
\hline \multicolumn{2}{|l|}{ Coronary artery calcium score groups } \\
\hline None $(=0)$ & $753(51.1 \%)$ \\
\hline Low/moderate (1-399) & $567(38.5 \%)$ \\
\hline High $(\geq 400)$ & $154(10.4 \%)$ \\
\hline \multicolumn{2}{|l|}{ Cardiac CT angiography $\ddagger(n=1470)$} \\
\hline \multicolumn{2}{|l|}{ Coronary artery disease severity } \\
\hline Non (stenosis $0 \%$ and $\mathrm{CACS}=0$ ) & $702(47.8 \%)$ \\
\hline Mild (stenosis $0 \%-29 \%$ ) & $306(20.8 \%)$ \\
\hline Moderate (stenosis 30\%-49\%) & $112(7.6 \%)$ \\
\hline Severe (stenosis $50 \%-100 \%$ ) & $350(23.8 \%)$ \\
\hline \multicolumn{2}{|c|}{ Invasive coronary angiography (QCA, anatomic disease) $\ddagger(n=325)$} \\
\hline \multicolumn{2}{|l|}{ Coronary artery disease severity } \\
\hline Mild stenosis (0\%-29\%) & $74(5.1 \%)$ \\
\hline Moderate stenosis ( $30 \%-49 \%)$ & $98(6.8 \%)$ \\
\hline $\begin{array}{l}\text { Severe stenosis ( } 50 \%-100 \% \text {, anatomically obstructive } \\
\text { stenosis) }\end{array}$ & $153(10.6 \%)$ \\
\hline \multicolumn{2}{|l|}{ Vessel disease } \\
\hline Left main coronary artery & $7(0.05 \%)$ \\
\hline Left anterior descending coronary artery & $96(6.6 \%)$ \\
\hline Left circumflex coronary artery & $53(3.7 \%)$ \\
\hline Right coronary artery & $67(4.6 \%)$ \\
\hline \multicolumn{2}{|l|}{ Coronary vessel disease } \\
\hline One-vessel disease & $94(6.5 \%)$ \\
\hline
\end{tabular}




\begin{tabular}{|c|c|}
\hline Two-vessel disease & $42(2.9 \%)$ \\
\hline Three-vessel disease or LM disease & $17(1.2 \%)$ \\
\hline \multicolumn{2}{|c|}{ Invasive coronary angiography (FFR, haemodynamic disease) $)(n=325)$} \\
\hline \multicolumn{2}{|l|}{ Coronary artery disease severity } \\
\hline Non-severe stenosis (FFR $>0.8$ or diameter stenosis $<30 \%$ ) & $180(12.4 \%)$ \\
\hline $\begin{array}{l}\text { Severe stenosis (FFR }<0.8) \text {, haemodynamically obstructive } \\
\text { stenosis }\end{array}$ & $145(10.0 \%)$ \\
\hline \multicolumn{2}{|l|}{ Vessel disease } \\
\hline Left main coronary artery & $7(0.05 \%)$ \\
\hline Left anterior descending coronary artery & $117(8.1 \%)$ \\
\hline Left circumflex coronary artery & $41(2.8 \%)$ \\
\hline Right coronary artery & $55(3.8 \%)$ \\
\hline \multicolumn{2}{|l|}{ Coronary vessel disease } \\
\hline One-vessel disease & $88(6.1 \%)$ \\
\hline Two-vessel disease & $39(2.7 \%)$ \\
\hline Three-vessel disease or left main coronary artery disease & $18(1.2 \%)$ \\
\hline \multicolumn{2}{|c|}{$\begin{array}{l}\text { Values are } \mathrm{n}(\%) \text { or mean } \pm \mathrm{SD} \text { or median }(\mathrm{IQR}) \text {. } \\
{ }^{*} \text { Coronary artery disease among first-degree relatives aged less than } 60 \text { years. } \\
\text { tMean heart rate at the time of CAD-score measurement was } 54 \pm 7 \mathrm{bpm} \text { and at the } \\
\text { time of CTA was } 56 \pm 7 \mathrm{bpm} \text {. } \\
\text { \$Cardiac CTA data were missing in four patients and ICA data were missing in } 25 \\
\text { patients with an indication to ICA due to severe CAD at cardiac CTA. } \\
\text { CAD, coronary artery disease; CCS, Canadian Cardiovascular Society; } \\
\text { CTA, CT angiography; FFR, fractional flow reserve; ICA, Invasive coronary } \\
\text { angiography; QCA, quantitative coronary angiography. }\end{array}$} \\
\hline
\end{tabular}

estimate $(r=0.01, P=0.98)$. There was a statistically significant stepwise increase in CAD-score $\mathrm{V} 3$ with increasing number of diseased vessels. There was no relation between CAD-score V3 and the anatomical location of the stenosis (table 3 ).

The diagnostic accuracy for the training $(n=593)$ versus the validation $(n=1082)$ cohort is summarised in online supplementary table S2.

Table 2 features the CAD-score V3 diagnostic accuracy with both anatomically and haemodynamically obstructive stenosis as references. The CAD-score V3 for diagnosing anatomically obstructive stenosis had an AUC of $72.4 \%$ (95\% CI $68.0 \%$ to $76.8 \%)$. This was significantly higher than the AUC of both the DF score $65.9 \%(\mathrm{P}<0.01)$ and the CAD-score V2 58.1\% $(\mathrm{P}<0.001)$, and the acoustic part of the CAD-score V3 63.3\% $(\mathrm{P}<0.001)$ when analysed in the full cohort. The AUCs of these algorithms for haemodynamically obstructive stenosis with FFR as a reference are illustrated in figure 4. Frequency plot, $2 \times 2$ table, and sensitivity and specificity curves with haemodynamically obstructive stenosis as reference are illustrated in figure 5. In total, 27 patients had false-negative CAD-scores and of these, $48.1 \%$ were men, mean age was 52.7 years, mean body mass index was 27.1, and mean left ventricular ejection fraction was $60.4 \%$.

\section{DISCUSSION}

In this large, first-of-its-kind prospective trial, we evaluated a novel acoustic technique for primary risk stratification and rule-out of obstructive CAD. We studied a cohort of symptomatic patients with low to intermediate risk of CAD referred to cardiac CTA. This patient cohort represents a relevant clinical setting where the use of an accurate rule-out device could have clinical impact. All patients in the trial had either a cardiac CTA or an ICA to rule out coronary stenosis. Furthermore, suspected stenosis at ICA was verified with both core lab QCA and invasive FFR.
Table 2 Diagnostic accuracy of CAD-score with a cut-off of $>20$ according to a reference of $(A)$ anatomically obstructive stenosis diagnosed with core lab QCA and (B) haemodynamically obstructive stenosis diagnosed with invasive FFR as reference

\begin{tabular}{|c|c|c|}
\hline & CAD-score Version 2 & CAD-score Version 3 \\
\hline \multicolumn{3}{|c|}{ A. Anatomically obstructive stenosis } \\
\hline Number of patients & 1450 & 1437 \\
\hline True positive & 100 & 119 \\
\hline False positive & 720 & 606 \\
\hline True negative & 577 & 683 \\
\hline False negative & 53 & 29 \\
\hline \multicolumn{3}{|l|}{ Accuracy analysis } \\
\hline Sensitivity & $65.4(95 \% \mathrm{Cl} 57.3$ to 72.9$)$ & $80.4(95 \% \mathrm{Cl} 73.1$ to 86.5$)$ \\
\hline Specificity & $44.5(95 \% \mathrm{Cl} 41.8$ to 47.2$)$ & $53.0(95 \% \mathrm{Cl} 50.2$ to 55.7$)$ \\
\hline PPV & $12.2(95 \% \mathrm{Cl} 10.0$ to 14.6$)$ & $16.4(95 \% \mathrm{Cl} 13.8$ to 19.3$)$ \\
\hline NPV & $91.6(95 \% \mathrm{Cl} 89.1$ to 93.6$)$ & $95.9(95 \% \mathrm{Cl} 94.2$ to 97.3$)$ \\
\hline PLR & $1.18(95 \% \mathrm{Cl} 1.04$ to 1.33$)$ & $1.71(95 \% \mathrm{Cl} 1.56$ to 1.88$)$ \\
\hline NLR & $0.78(95 \% \mathrm{Cl} 0.62$ to 0.98$)$ & 0.37 ( $95 \% \mathrm{Cl} 0.27$ to 0.51$)$ \\
\hline AUC & $58.1(95 \% \mathrm{Cl} 53.6$ to 62.6$)$ & $72.4(95 \% \mathrm{Cl} 68.0$ to 76.8$)$ \\
\hline \multicolumn{3}{|c|}{ B. Haemodynamically obstructive stenosis } \\
\hline Number of patients & 1450 & 1437 \\
\hline True positive & 92 & 113 \\
\hline False positive & 728 & 612 \\
\hline True negative & 577 & 685 \\
\hline False negative & 53 & 27 \\
\hline \multicolumn{3}{|l|}{ Accuracy analysis } \\
\hline Sensitivity & $63.4(95 \% \mathrm{Cl} 55.1$ to 71.3$)$ & 80.7 (95\% Cl 73.2 to 86.9$)$ \\
\hline Specificity & $44.2(95 \% \mathrm{Cl} 41.5$ to 47.0$)$ & $52.8(95 \% \mathrm{Cl} 50.0$ to 55.6$)$ \\
\hline PPV & $11.2(95 \%$ Cl 9.1 to 13.6$)$ & $15.6(95 \% \mathrm{Cl} 13.0$ to 18.4$)$ \\
\hline NPV & $91.6(95 \% \mathrm{Cl} 89.1$ to 93.6$)$ & $96.2(95 \% \mathrm{Cl} 94.5$ to 97.5$)$ \\
\hline PLR & $1.14(95 \% \mathrm{Cl} 1.00$ to 1.30$)$ & $1.71(95 \% \mathrm{Cl} 1.56$ to 1.89$)$ \\
\hline NLR & $0.83(95 \% \mathrm{Cl} 0.66$ to 1.03$)$ & $0.36(95 \% \mathrm{Cl} 0.25$ to 0.50$)$ \\
\hline AUC & $56.8(95 \% \mathrm{Cl} 51.8$ to 61.7$)$ & 71.3 (95\% Cl 66.8 to 75.8$)$ \\
\hline
\end{tabular}

AUC, area under the curve; FFR, fractional flow reserve; NLR, negative likelihood ratio; NPV, negative predictive value; PLR, positive likelihood ratio; PPV, positive predictive value; QCA, quantitative coronary angiography.

\section{CAD-score V2}

The isolated acoustic CAD-score V2 had a significantly lower diagnostic accuracy for predicting coronary stenosis than a DF score which is recommended in clinical guidelines. However, both the CAD-score V2 and the DF score performed poorly in this low-risk cohort of patients referred to cardiac CTA (prevalence of $\mathrm{CAD}=10 \%$ ) compared with a previous study with a moderate/high-risk cohort consisting of patients referred to either cardiac CTA or ICA (prevalence of CAD $=28 \%$ ). ${ }^{4}$ Hence, present versus previously published AUCs with anatomically obstructive stenosis as reference were: CAD-score V2: 58\% vs $72 \%$; and DF score: $65 \%$ vs $79 \%$. The CAD-score V2 was developed using 228 primarily moderate/high-risk patients and previously only validated by cross validation. These facts might have induced an overfit of the algorithm to cohorts with a higher CAD prevalence.

\section{CAD-score V3}

Data from the updated CAD-score V3 are presented from both the training and the validation cohorts owing to similar accuracy between the two cohorts. A moderate association between CAD-score V3 and CACS was detected in this study; we demonstrated a significant difference in the mean CAD-score between the CACS groups. The acoustic risk score as determined by either 
A

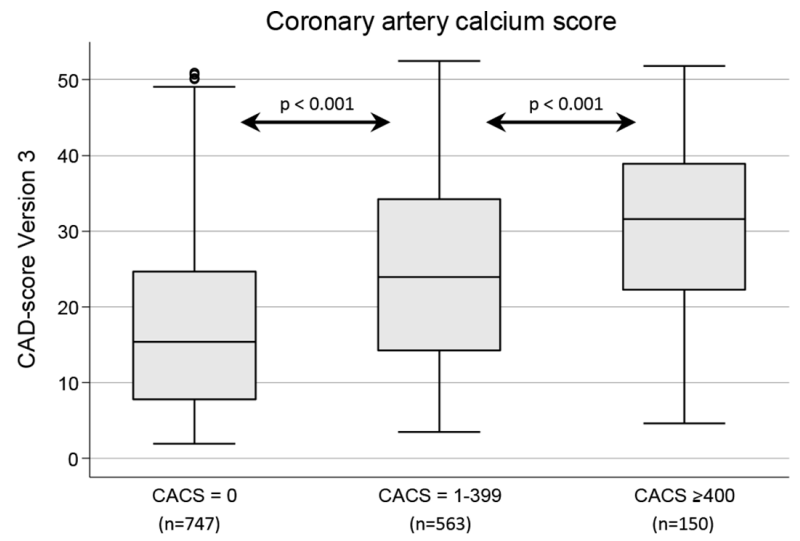

B

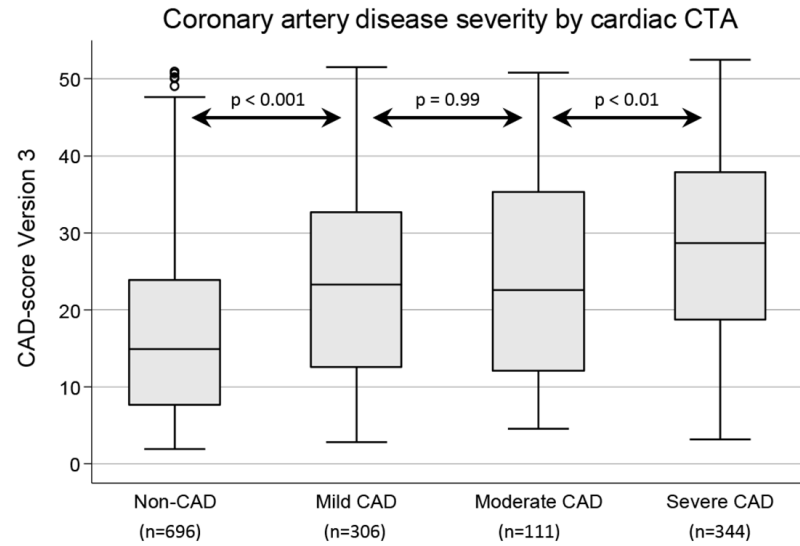

C

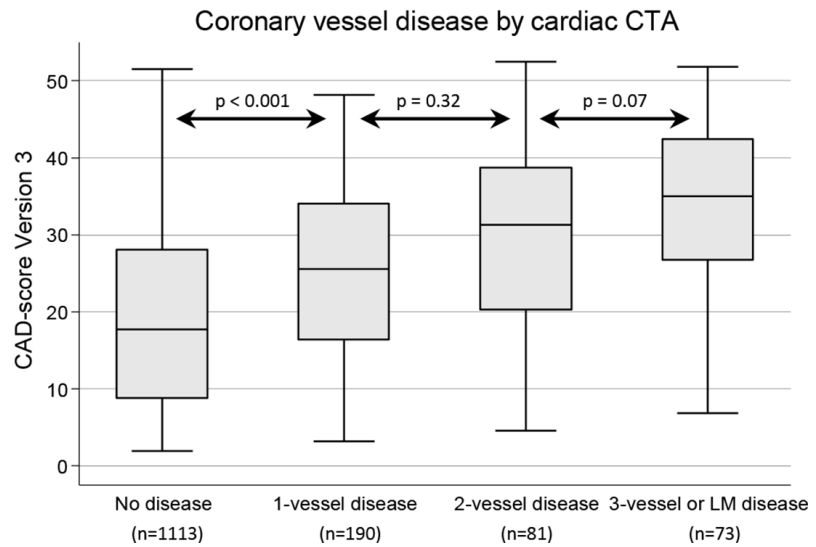

Figure 3 CAD-score Version 3 divided by: (A) coronary artery calcium score groups; (B) CAD disease severity defined by cardiac CTA; (C) haemodynamically obstructive coronary vessel disease by cardiac CTA. Box plot illustrated median, IQR and adjacent values. CACS, coronary artery calcium score; CAD, coronary artery disease; $C T A, C T$ angiography; $\mathrm{LM}$, left main.

the CADScor®System device or the Cardiac Sonospectrographic Analyzer (CSA, SonoMedica, Vienna, Virginia) has previously shown weak correlation with CACS. ${ }^{4}{ }^{10}$ Hence, the inclusion of risk factors (gender, age and hypertension) to the algorithm increased the correlation with CACS, which previously demonstrated high prognostic value. ${ }^{17} 18$

Comparable accuracy was detected when using QCA and FFR as reference standard of disease despite the fact that the CAD-score was optimised in order to detect stenosis accessed by QCA. Compared with the DF score, the CAD-score V3 was
Table 3 Relation between haemodynamically obstructive disease distribution at ICA and mean CAD-score Version $3(n=320)$

\begin{tabular}{lll}
\hline All patients & $\begin{array}{l}\text { CAD-score } \\
\text { Version 3 }\end{array}$ & P value \\
\hline No disease & $26.3 \pm 12.0$ & $<0.05$ \\
One-vessel disease & $28.7 \pm 11.7$ & \\
Two-vessel disease & $31.6 \pm 11.5$ & \\
Three-vessel or left main coronary artery disease & $32.5 \pm 13.9$ & \\
Left main coronary artery & $32.8 \pm 16.4$ & 0.97 \\
Left anterior descending coronary artery & $30.6 \pm 12.0$ & \\
Left circumflex coronary artery & $31.8 \pm 11.4$ & \\
Right coronary artery & $30.3 \pm 12.3$ & \\
Patients with one-vessel disease* & & \\
Left main coronary artery & NA & 0.50 \\
Left anterior descending coronary artery & $29.4 \pm 11.6$ & \\
Left circumflex coronary artery & $24.6 \pm 6.8$ & \\
Right coronary artery & $26.7 \pm 12.5$ & \\
\hline
\end{tabular}

${ }^{*}$ The number of patients with haemodynamically obstructive one-vessel disease at ICA was 84 in total: left anterior descending coronary artery $(n=63)$, left circumflex coronary artery $(n=4)$ and right coronary artery $(n=17)$. $\mathrm{NA}$, not applicable.

more accurate in detecting anatomically obstructive $\mathrm{CAD}$, and a trend was seen towards a high accuracy for haemodynamically obstructive CAD. In addition, CAD-score V3 increased with the number of affected coronary vessels by cardiac CTA and ICA. Importantly, CAD-score V3 was not related to the location of the stenosis.

\section{Acoustic recording and diagnostic algorithm}

Several acoustic methods are currently being tested for detection of coronary stenosis. ${ }^{8}$ The recording system used in this study is mounted by a single-use adhesive patch at the fourth intercostal space, and recordings are performed automatically during four short breath holds over a 3 min recording period. The result is subsequently calculated and presented on the device display. Other systems use multiple record positions, handheld and elastic strap
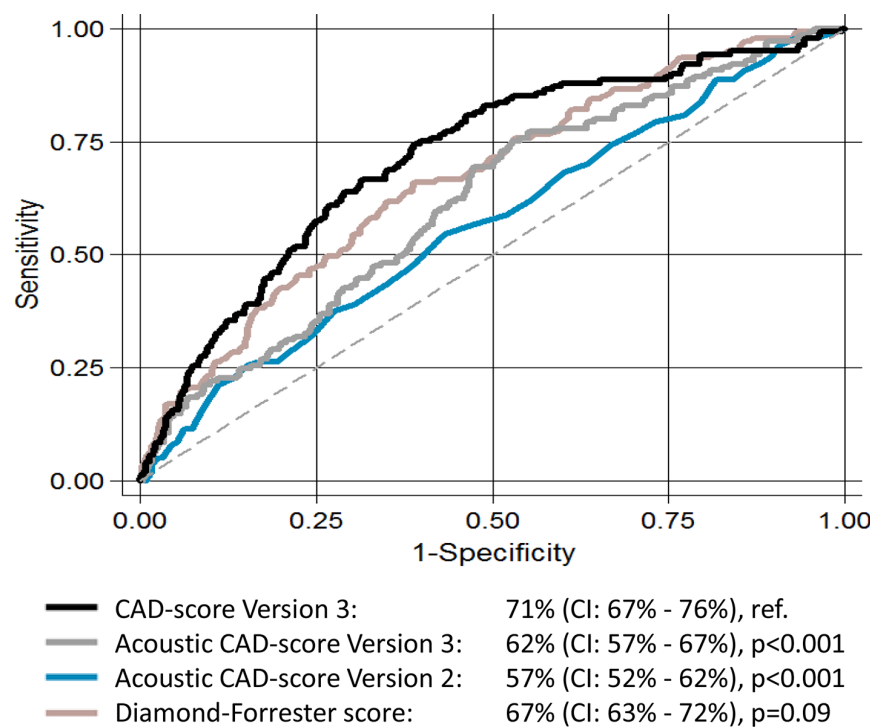

Figure 4 The area under the receiver operating characteristic curve for Diamond-Forrester score, acoustic CAD-score Versions 2 and 3, and CAD-score Version 3 with haemodynamically obstructive stenosis diagnosed as reference. 

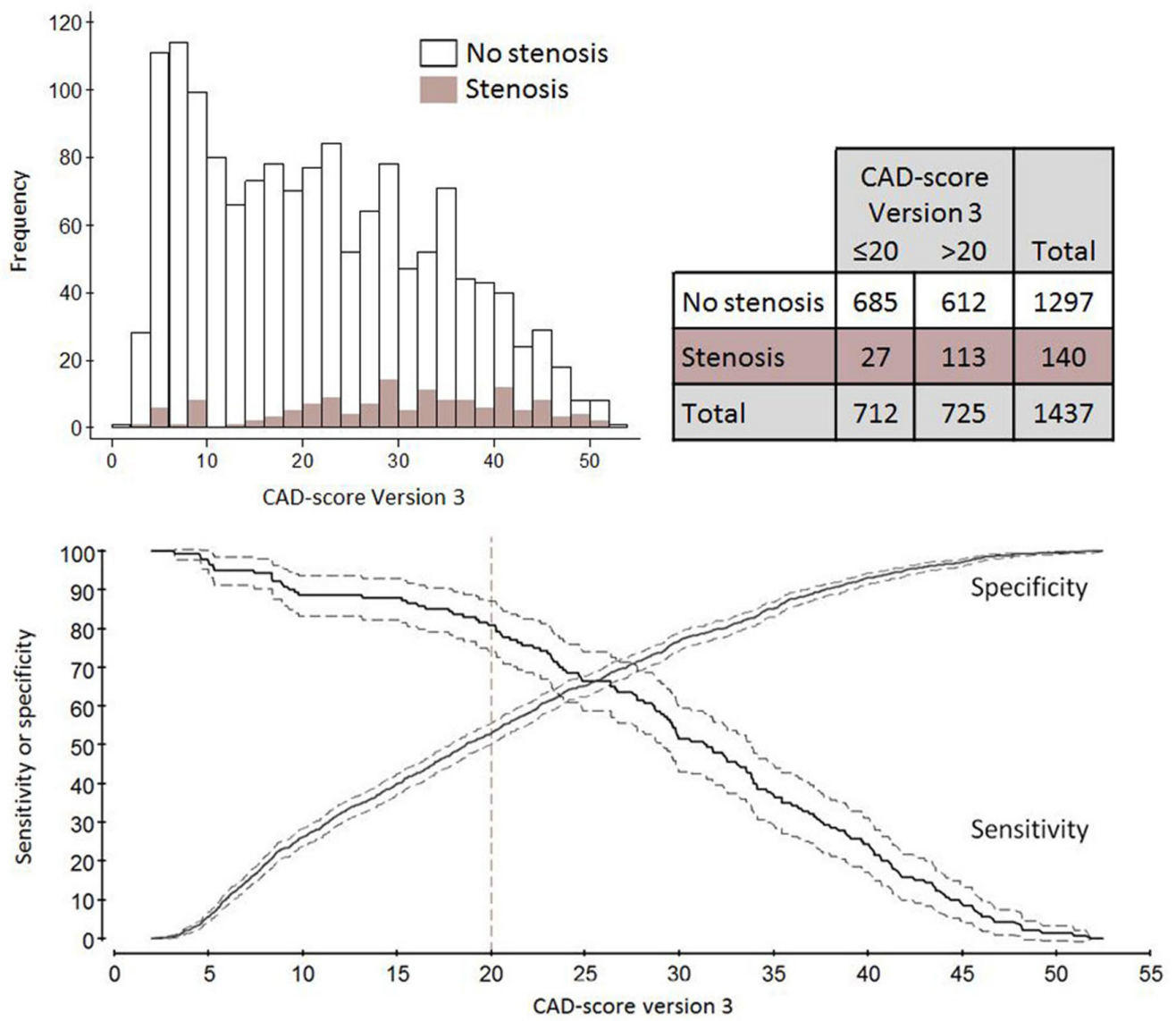

Figure 5 Plots of CAD-score Version 3 accuracy of haemodynamically obstructive coronary stenosis diagnosed with fractional flow reserve (FFR) as reference. Illustrated are a frequency plot, $2 \times 2$ table according to a binary CAD-score cut-off $>20$, and sensitivity and specificity curves with $95 \%$ CI bands shown.

attachment, and one system performs records simultaneously with an electrocardiographic signal. The methodological differences have inherent limitations and advantages. The adhesive patches may reduce microphone noise and inter-recording variability. Single versus multiple recording positions may limit the ability to differentiate between stenosis locations. Individual body compositions may be crucial for correct placement of the recording devices and sound quality. Similarly, there are major differences in software algorithms and which spectral frequency ranges are included. ${ }^{8}$ These differences need to be highlighted to further optimise both the equipment and the algorithm. Finally, studies of how patient characteristics, coronary plaque length, location, compositions and flow impact the acoustic patterns are lacking and insights from such studies could potentially enhance system performance.

The latest UK NICE (National Institute for Health and Care Excellence) guideline update CG95: Chest pain of recent onset, includes the acoustic device as a clinically relevant prediction model with an AUC ranging from $72 \%$ to $82 \%$, alone or in combination with the DF score.

Comparing the sensitivity of this acoustic device with the sensitivity of other large-scale, presently used techniques is also relevant; for example, exercise ECG (sensitivity 45\%-50\%), exercise stress echocardiography (sensitivity 80\%-85\%) and exercise stress SPECT $(73 \%-92 \%){ }^{12}$ Thus, the above-mentioned techniques have false negative rates above or similar to those reported in the present study. Nonetheless, these other diagnostic modalities have previously all been proven to hold substantial prognostic value, which still needs to be demonstrated for acoustic rule-out algorithms.
The health economic impact of acoustic rule-out of patients with symptoms suggestive of obstructive CAD could be substantial. As mentioned above, the acoustic system seems to perform similarly to other established techniques in ruling out $\mathrm{CAD}$, but its use comes at a potentially much lower cost.

\section{Limitations}

The cohort was almost exclusively Caucasian (99\%), and with a documented low to intermediate prevalence of CAD. Thus,

\section{Key messages}

What is already known on this subject?

Recent advances in sound sensor technology, analytic power and data filtering have enabled the use of acoustic detection to diagnose intracoronary turbulence due to obstructive coronary artery disease (CAD).

What might this study add?

In this large, first-of-its-kind prospective trial, we demonstrated that acoustic detection of CAD enables risk stratification in patients with suspected CAD.

How might this impact on clinical practice? The negative predictive value of this device suggests that it may be used as a rule-out modality, which is highly relevant in daily clinical practice. 
generalisation to other populations with other ethnic backgrounds, higher prevalence and other healthcare environments may not be possible. Higher CAD-scores were observed for a low number of patients with heart valve disease, however only when adding the clinical risk factors, indicating that patients with heart valve disease are diagnosed accurately. Finally, the development of CAD-score $\mathrm{V} 3$ required splitting of the cohort into a training and a validation cohort, which could have overfitted the results in the total cohort analysis.

\section{CONCLUSION}

The sound-based recording device, the CADScorSystem, enables risk stratification in patients with suspected CAD. With an NPV of $96 \%$, this new acoustic rule-out system could potentially supplement clinical assessment to guide decisions on the need for further investigation and thereby reduce the demand for more advanced and costly diagnostic modalities.

Contributors Substantial contribution to conception and design: SW, LN, SES, NRH, HEB and MB. Collection of data: SW, LN, JSW, LDR, LLK, LHM, JKJ and LF. Analysis and interpretation of data: SW, LN, SES, JSW, BSL, JJS, EHC, HEB and MB. All authors have worked on drafting the article or revising it critically and approved the final version.

Funding The research reported here is partly financed by The Danish Heart Foundation (grant no. 15-R99-A5837-22920), The Hede Nielsen Foundation, and by an unrestricted institutional research grant from Acarix A/S.

Competing interests The current research is financed partly by Acarix A/S by an unrestricted grant. SES is a minor shareholder and part-time consultant in Acarix A/S. BSL is an industrial student at Acarix A/S. MB is part of the Acarix A/S advisory board. MB and SW received an unrestricted institutional research grant from Acarix A/S.

Ethics approval The study was approved by The Central Denmark Region Committees on Health Research Ethics and The Danish Data Protection Agency.

Provenance and peer review Not commissioned; externally peer reviewed.

Open Access This is an Open Access article distributed in accordance with the Creative Commons Attribution Non Commercial (CC BY-NC 4.0) license, which permits others to distribute, remix, adapt, build upon this work non-commercially, and license their derivative works on different terms, provided the original work is properly cited and the use is non-commercial. See: http://creativecommons.org/ licenses/by-nc/4.0/

(c) Article author(s) (or their employer(s) unless otherwise stated in the text of the article) 2018. All rights reserved. No commercial use is permitted unless otherwise expressly granted.

\section{REFERENCES}

1 Levitt K, Guo H, Wijeysundera HC, et al. Predictors of normal coronary arteries at coronary angiography. Am Heart J 2013;166:694-700.

2 Nielsen LH, Nørgaard BL, Tilsted HH, et al. The Western Denmark cardiac computed tomography registry: a review and validation study. Clin Epidemiol 2015;7:53-64

3 Patel MR, Peterson ED, Dai D, et al. Low diagnostic yield of elective coronary angiography. N Eng/ J Med 2010;362:886-95.

4 Winther S, Schmidt SE, Holm NR, et al. Diagnosing coronary artery disease by sound analysis from coronary stenosis induced turbulent blood flow: diagnostic performance in patients with stable angina pectoris. Int J Cardiovasc Imaging 2016:32:235-45.

5 Schmidt SE, Holst-Hansen C, Hansen J, et al. Acoustic features for the identification of coronary artery disease. IEEE Trans Biomed Eng 2015;62:2611-9.

6 Schmidt SE, Hansen J, Zimmermann $\mathrm{H}$, et al. Coronary artery disease and low frequency heart sound signatures. Comput Cardiol 2011;38:481-4.

7 Wang JZ, Tie B, Welkowitz W, et al. Modeling sound generation in stenosed coronary arteries. IEEE Trans Biomed Eng 1990;37:1087-94.

8 Thomas JL, Winther S, Wilson RF, et al. A novel approach to diagnosing coronary artery disease: acoustic detection of coronary turbulence. Int I Cardiovasc Imaging 2017;33:129-36

9 Semmlow J, Rahalkar K. Acoustic detection of coronary artery disease. Annu Rev Biomed Eng 2007;9:449-69.

10 Makaryus AN, Makaryus JN, Figgatt A, et al. Utility of an advanced digital electronic stethoscope in the diagnosis of coronary artery disease compared with coronary computed tomographic angiography. Am J Cardiol 2013;111:786-92.

11 Azimpour F, Caldwell E, Tawfik P, et al. Audible coronary artery stenosis. Am J Med 2016;129:515-21.

12 Montalescot G, Sechtem U, Achenbach S, et al. 2013 ESC guidelines on the management of stable coronary artery disease: the Task Force on the management of stable coronary artery disease of the European Society of Cardiology. Eur Heart J 2013;34:2949-3003.

13 Genders TS, Steyerberg EW, Alkadhi H, et al. A clinical prediction rule for the diagnosis of coronary artery disease: validation, updating, and extension. Eur Heart J 2011;32:1316-30.

14 Nissen L, Winther S, Isaksen C, et al. Danish study of Non-Invasive testing in Coronary Artery Disease (Dan-NICAD): study protocol for a randomised controlled trial. Trials 2016;17:262.

15 Schmidt SE, Holst-Hansen C, Graff C, et al. Segmentation of heart sound recordings by a duration-dependent hidden Markov model. Physiol Meas 2010;31:513-29.

16 DeLong ER, DeLong DM, Clarke-Pearson DL. Comparing the areas under two or more correlated receiver operating characteristic curves: a nonparametric approach. Biometrics 1988;44:837-45.

17 Knapper JT, Khosa F, Blaha MJ, et al. Coronary calcium scoring for long-term mortality prediction in patients with and without a family history of coronary disease. Heart 2016;102:204-8.

18 Chang SM, Nabi F, Xu J, et al. Value of CACS compared with ETT and myocardial perfusion imaging for predicting long-term cardiac outcome in asymptomatic and symptomatic patients at low risk for coronary disease: clinical implications in a multimodality imaging world. JACC Cardiovasc Imaging 2015;8:134-44. 